\title{
scNetViz: from single cells to networks using Cytoscape
}

\section{[version 1; peer review: 2 approved]}

\author{
Krishna Choudhary'1, Elaine C. Meng2', J. Javier Diaz-Mejia(iD2-5, \\ Gary D. Bader (D)3,4,6,7, Alexander R. Pico (D)1, John H. Morris (iD)2 \\ ${ }^{1}$ Institute of Data Science and Biotechnology, Gladstone Institutes, San Francisco, California, 94158, USA \\ 2Department of Pharmaceutical Chemistry, University of California San Francisco, San Francisco, California, 94143, USA \\ 3 Princess Margaret Cancer Centre, University Health Network, Toronto, Ontario, M5G 2M9, Canada \\ ${ }^{4}$ The Donnelly Centre, University of Toronto, Toronto, Ontario, M5S 3E1, Canada \\ ${ }^{5}$ Phenomic AI, Toronto, Ontario, M5G 0B7, Canada \\ ${ }^{6}$ Department of Molecular Genetics, University of Toronto, Toronto, Ontario, M5G 1A8, Canada \\ ${ }^{7}$ Department of Computer Science, University of Toronto, Toronto, Ontario, M5T 3A1, Canada
}

V1 First published: 07 Jun 2021, 10:448

https://doi.org/10.12688/f1000research.52460.1

Latest published: 07 Jun 2021, 10:448

https://doi.org/10.12688/f1000research.52460.1

\section{Abstract}

Single-cell RNA-sequencing (scRNA-seq) has revolutionized molecular biology and medicine by enabling high-throughput studies of cellular heterogeneity in diverse tissues. Applying network biology approaches to scRNA-seq data can provide useful insights into genes driving heterogeneous cell-type compositions of tissues. Here, we present scNetViz - a Cytoscape app to aid biological interpretation of cell clusters in scRNA-seq data using network analysis. scNetViz calculates the differential expression of each gene across clusters and then creates a cluster-specific gene functional interaction network between the significantly differentially expressed genes for further analysis, such as pathway enrichment analysis. To automate a complete data analysis workflow, scNetViz integrates parts of the Scanpy software, which is a popular Python package for scRNA-seq data analysis, with Cytoscape apps such as stringApp, cyPlot, and enhancedGraphics. We describe our implementation of methods for accessing data from public single cell atlas projects, differential expression analysis, visualization, and automation. scNetViz enables users to analyze data from public atlases or their own experiments, which we illustrate with two use cases. Analysis can be performed via the Cytoscape GUI or CyREST programming interface using R (RCy3) or Python (py4cytoscape).

\section{Keywords}

scRNA-seq, Single cell, Expression analysis, Cytoscape, App, Network biology

\section{Open Peer Review \\ Approval Status \\ 1 2 \\ version 1 \\ 07 Jun 2021

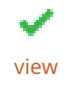 \\ view \\ 1. Luca Pinello, Harvard Medical School, \\ Boston, USA \\ 2. Kenji Kamimoto (D), Washington University \\ School of Medicine in St. Louis, St. Louis, USA \\ Any reports and responses or comments on the article can be found at the end of the article.}


This article is included in the Bioinformatics

gateway.

This article is included in the Cell \& Molecular

Biology gateway.

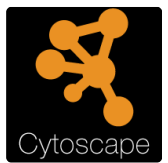

This article is included in the Cytoscape

gateway.

Corresponding author: John H. Morris (scooter@cgl.ucsf.edu)

Author roles: Choudhary K: Validation, Visualization, Writing - Original Draft Preparation, Writing - Review \& Editing; Meng EC: Conceptualization, Validation, Writing - Original Draft Preparation, Writing - Review \& Editing; Diaz-Mejia JJ: Conceptualization, Writing Review \& Editing; Bader GD: Conceptualization, Funding Acquisition, Methodology, Writing - Review \& Editing; Pico AR:

Conceptualization, Funding Acquisition, Methodology, Project Administration, Resources, Supervision, Validation, Writing - Original Draft Preparation, Writing - Review \& Editing; Morris JH: Conceptualization, Funding Acquisition, Methodology, Project Administration, Resources, Software, Supervision, Validation, Writing - Original Draft Preparation, Writing - Review \& Editing

Competing interests: No competing interests were disclosed.

Grant information: JHM, ECM, JJDM and ARP are supported by grant number 2018-183120 from the Chan Zuckerberg Initiative DAF, an advised fund of the Silicon Valley Community Foundation. JHM, GDB, KC and ARP are supported by NIGMS P41GM103504 for the National Resource for Network Biology.

The funders had no role in study design, data collection and analysis, decision to publish, or preparation of the manuscript.

Copyright: $\odot 2021$ Choudhary $\mathrm{K}$ et al. This is an open access article distributed under the terms of the Creative Commons Attribution License, which permits unrestricted use, distribution, and reproduction in any medium, provided the original work is properly cited.

How to cite this article: Choudhary K, Meng EC, Diaz-Mejia JJ et al. scNetViz: from single cells to networks using Cytoscape [version 1; peer review: 2 approved] F1000Research 2021, 10:448 https://doi.org/10.12688/f1000research.52460.1

First published: 07 Jun 2021, 10:448 https://doi.org/10.12688/f1000research.52460.1 


\section{Introduction}

Single-cell RNA sequencing (scRNA-seq) has yielded significant insights into mechanisms regulating diverse biological systems. ${ }^{1}$ This technology captures transcriptome-wide expression profiles of single cells, which can be used to cluster cells and identify the biological features that distinguish the clusters. ${ }^{2}$ With continued technological advances and cost efficiencies, scRNA-seq is becoming increasingly common and new data are being generated at a rapid pace. Global efforts such as the Human Cell Atlas, ${ }^{3}$ which aims to map a healthy human, and the EMBL-EBI Single Cell Expression Atlas, ${ }^{4}$ which organizes published datasets across multiple species and provides access to results from standardized analyses, are growing rapidly. To study cell types in scRNA-seq data, it is useful to compare cell clusters to identify celltype-specific gene expression markers and genes associated with a phenotype. ${ }^{2,5}$ Such analysis typically provides a ranking of genes based on the statistical significance of differential expression. Besides this, applying network biology approaches can yield helpful insights for biomedical applications. ${ }^{6,7,8,9}$ In this direction, some methods enable inference of cell-type-specific regulatory networks from the experimental data. ${ }^{10,11,12,13}$ In contrast, another approach is to filter gene networks from independent experiments or public databases using single-cell data for cell-type-specific insights. ${ }^{7,14}$

Public databases such as the STRING database ${ }^{15}$ contain high-quality and well-organized information about published interaction networks. Here, we describe $s c \mathrm{NetViz}$ - an interactive desktop Java application that harnesses the extensive network visualization and analysis capabilities of Cytoscape for single-cell data analysis. ${ }^{16,17}$ scNetViz takes a network filtering approach as mentioned above. It enables users to load their own scRNA-seq data or pull such data from public atlases, cluster cells, run differential gene expression analysis, perform dimensionality reduction of expression matrices for data visualization, generate heatmaps and violin plots of gene expression data, and examine the associated gene interaction networks from the STRING database. Below, we describe the methods implemented in scNetViz, provide two use cases to illustrate the user interface and programmatic access using $\mathrm{R}$ and Python, and discuss plans for future development. scNetViz enables scientists who may not be experts in scripting to explore the data and to develop biological hypotheses. Further, it provides a convenient interface for integrating gene network information with scRNA-seq datasets, which can save time for researchers.

\section{Methods}

Overview

scNetViz consists of two main components, a Java-based Cytoscape app and a web service implemented on a compute cluster at the Resource for Biocomputing, Visualization and Informatics (RBVI) at the University of California, San Francisco. In addition, the app portion uses several other Cytoscape apps, including stringApp ${ }^{18}$ cyPlot,${ }^{19}$ and enhancedGraphics. ${ }^{20}$

The Cytoscape app provides the following functionality:

1. Acts as the graphical front-end for $s c N e t V i z$, including providing various dialogs for interactive users and commands for automation uses.

2. Manages all of the data that has been imported. This includes acting as a client for the Single Cell Expression Atlas ${ }^{4}$ and Human Cell Atlas $^{3}$ data portal REST services as well as providing users the option to import single cell expression matrices and category data (e.g. tissue, cluster information, experimental condition, etc.) from local files.

3. Calculates the differential gene expression for a chosen category (e.g. a specific clustering resolution or sample characteristic such as disease vs. non disease): a separate calculation for each group of cells (e.g. cluster) within that category vs. the set of all other cells (e.g. clusters) within that same category.

4. Fetches functional association networks from STRING for the protein products of significantly differentially expressed genes.

5. Interfaces with the RBVI web service described above to calculate cell plots based on $\mathrm{UMAP}^{21}$ or t-SNE. $^{22}$

6. Provides a Java-based implementation of t-SNE for smaller experiments (can be faster than the web service).

7. Interfaces with cyPlot, a Cytoscape app that displays various plots in the Cytoscape web browser, to visualize all UMAP and t-SNE embeddings as well as volcano plots and heatmaps.

8. Finally, it also interfaces with the RBVI web service to calculate clusters based on either the Louvain or Leiden algorithms as implemented in Scanpy. ${ }^{23}$ 
The RBVI web service component provides a simple wrapper around Scanpy that exposes REST endpoints for:

1. Calculating a UMAP embedding of an scRNA-seq matrix,

2. Calculating a tSNE embedding of an scRNA-seq matrix,

3. Calculating a Leiden clustering of an scRNA-seq matrix,

4. Calculating a Louvain clustering of an scRNA-seq matrix.

Matrices are passed as compressed MatrixMarket files ${ }^{24}$ and parameters may be provided to simplify and normalize the matrix in a pre-processing step, in addition to parameters related to the appropriate algorithm.

\section{Cytoscape app}

The Cytoscape App provides the main front-end, data management, and orchestration for scNetViz. Importantly, the app also

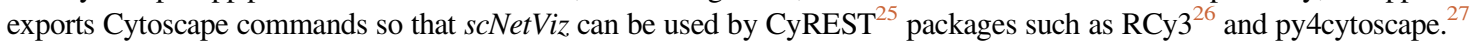
Below, we discuss five of the app functions: data access, differential gene expression calculation, plot generation, network creation, and automation via $\mathrm{R}$ and Python. The various user interface components are all implemented in Java Swing, use the Cytoscape Java Desktop API, and are shown in the use case descriptions.

\section{Data access}

scNetViz supports three types of data: cell by gene matrices (typically read in MatrixMarket format), experiment-level metadata including organism, number of cells in the experiment, and category-level metadata such as cell cluster assignments, disease state, cell type, tissue, ethnicity, which is typically in comma-separated value (CSV) format. scNetViz has a sources module, which includes submodules for each supported data source. A source module provides the tasks necessary to import data for an experiment or category, enumerates the available experiments and displays them. We have currently implemented support for three sources: EMBL-EBI's Single Cell Expression Atlas, the Human Cell Atlas data portal, and local files. The former two are public sources and are referred to as GXA and HCA, respectively, in scNetViz commands. They provide support for browsing experiments, while the local source provides methods to read category and experiment files. Since different category data might be in a different orientation (typically clusterings files are organized with the clusters in rows and cells in columns, but cell metadata and demographics are organized with the cells in rows and categorical information in the columns), we provided the ability to read in the CSV and transpose the resulting matrix, which may be needed to feed the data to downstream analysis steps.

To import data from the two public repositories, we rely on their published REST interfaces. The Single Cell Expression Atlas provided the simplest interface as specific endpoints for the matrices, clusters, and experimental-design:

- To enumerate all experiments: https://www.ebi.ac.uk/gxa/sc/json/experiments. This returns a JSON file that contains an entry for each experiment and significant metadata about the experiment.

- To import an experiment: https://www.ebi.ac.uk/gxa/sc/experiment $\wedge \%$ s/download/zip?fileType=normalised (where "\%s" is the accession identifier for the experiment). This returns a zip archive with the matrix, cell barcodes, and gene identifiers.

- To import the clusters provided: https://www.ebi.ac.uk/gxa/sc/experiment/\%s/download?fileType=cluster. This returns a CSV file of clustering results for various resolutions with an indication of the best resolution according to GXA.

- To import the experimental design: https://www.ebi.ac.uk/gxa/sc/experiment $/ \%$ s/download?fileType= experiment-design. This returns a CSV file that is transposed before being displayed.

Data access from the Human Cell Atlas recently transitioned to a new data coordination platform (DCP) API. scNetViz currently uses the "DCP1" API, and we will transition to "DCP2" in the near future. To access the DCP1 data:

- We can enumerate all experiments using the URL: https://service.azul.data.humancellatlas.org/index/projects? catalog=dcp 1 but, unlike the Single Cell Expression Atlas, the Human Cell Atlas contains raw data (e.g. Fastq and 
BAM files) as well as calculated matrices. To restrict our enumeration to include only matrix data, we need to include a filter, which is encoded as part of the URL. We use the following filter.

$\{$ "fileFormat":\{ "is":[ "matrix"] \} \}

It is encoded in the URL as using the "filters" query option: https://service.azul.data.humancellatlas.org/index/projects? catalog $=$ dcp $1 \backslash \&$ filters $=\{$ "fileFormat": $\{$ "is":["matrix"] $\}\}$. This query results in a JSON file that includes metadata about each experiment and a link to the expression matrix file itself. Because in the Human Cell Atlas a single "Project" may include multiple matrices, one for each tissue or organ that was investigated, we split those projects into independent experiments.

- To load the experiment data, we follow the URL provided by the experiment-level metadata. The result is a zip file that contains the matrix itself in MatrixMarket format, the gene identifiers, and a file that contains both the cell barcodes and categorical data for each cell.

\section{Differential expression calculation}

As shown below, the key function of $s c N e t V i z$ is to calculate the differential expression of each gene across categories and then use the highly differentially expressed genes (possible marker genes) to construct a gene functional interaction network. We calculate differential gene expression using Mann-Whitney U test and represent it as the $\log _{2}$ (fold change) where fold change is the ratio of the mean expression of a gene in a single group within a category vs. the mean expression of that gene in the comparison set of all other groups within that same category. For example, if the chosen category is a clustering with $\mathrm{K}=5$ classes, then each of the five classes is one group within the category. We provide two cutoff values to reduce noise in the resulting gene interaction network. First, a gene must be expressed in at least $10 \%$ of the cells in the group or $10 \%$ of the cells in the set of all other groups in the category. Second, we have a minimum $\log _{2}$ (fold change) cutoff that drops any changes that are beneath that threshold. All of this data is provided in a table that is created and provided to the user.

\section{Plot generation}

All of the plots provided by scNetViz are generated by the cyPlot Cytoscape App. ${ }^{19}$ cyPlot uses the built-in Cytoscape web browser and the plotly.js Javascript library to display plots. All of the data is passed to cyPlot using its automation interface. For example, below is the scNetViz method to create a violin plot.

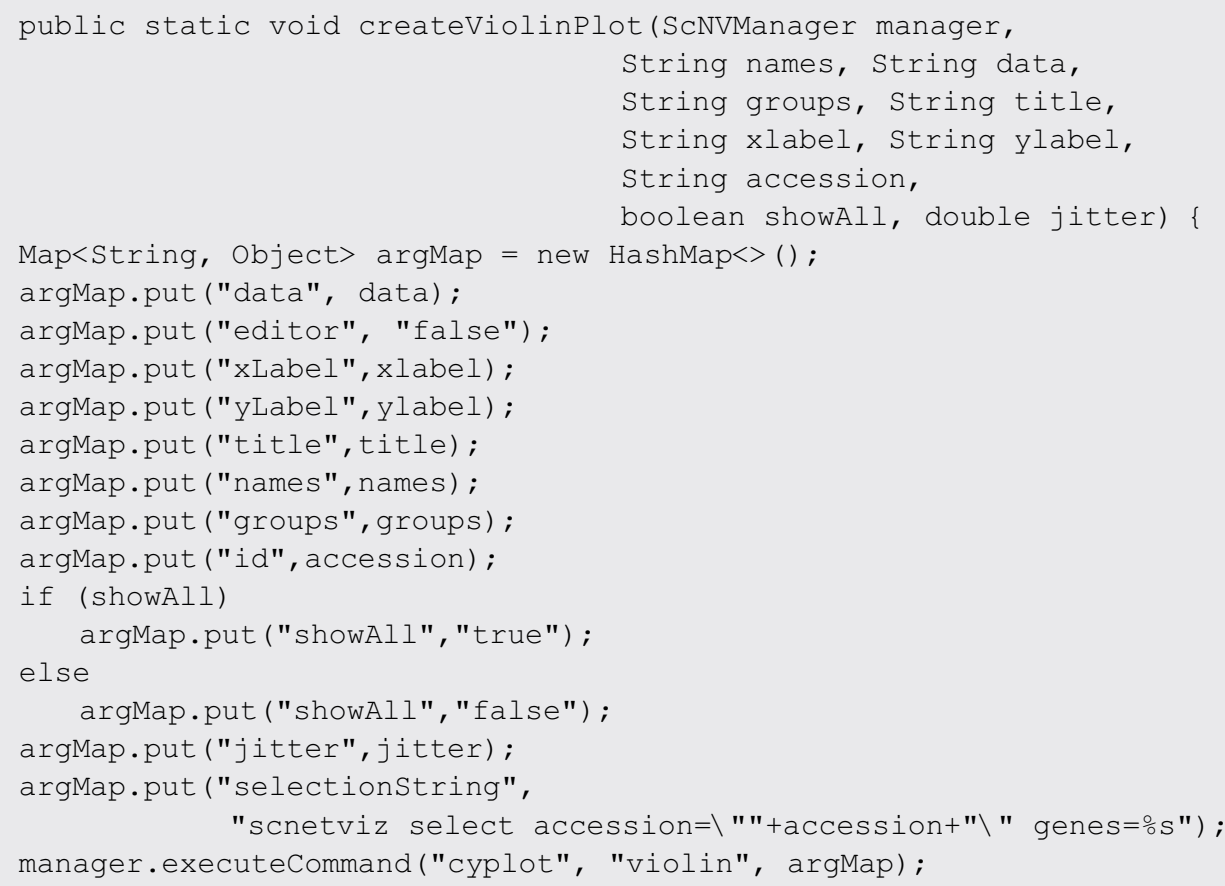


All of the arguments in the above code chunk are JSON strings that are consumed by cyPlot and passed on to the plotly.js library for display.

\section{Network creation}

Once the calculation of differential expression is complete, scNetViz can create networks to show the functional interactions among the proteins encoded by the differentially expressed genes. This proceeds in two steps. First, the STRING network of a user-specified number (default 200) of top differentially expressed genes for each group within the category is created by using the Cytoscape automation interface of the stringApp. The method that does this is:

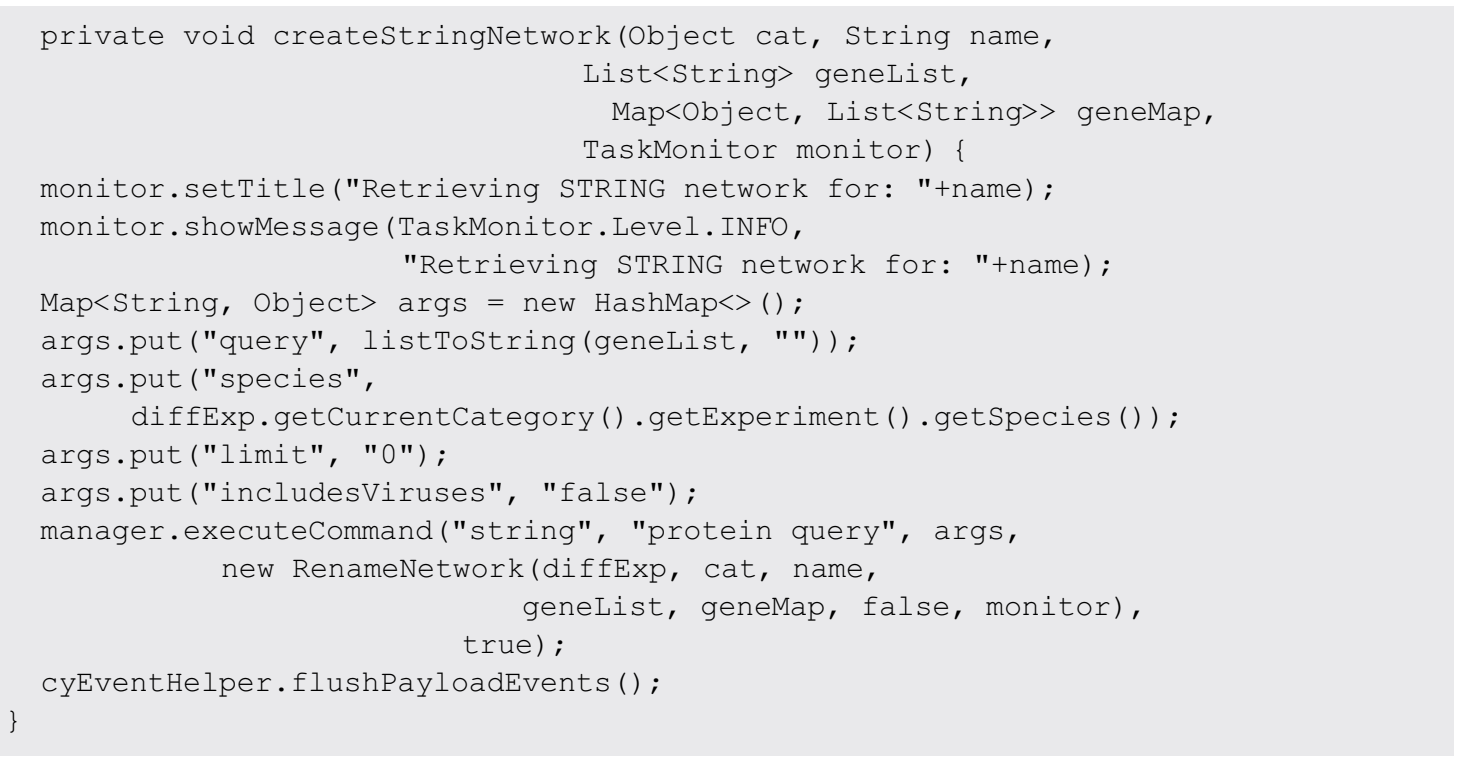

This creates the argument map and then calls the executeCommand method, which is a wrapper method that we implemented around the Cytoscape org.cytoscape.command.CommandExecutorTaskFactory. The stringApp queries STRING and creates the network. The second part of the process extracts significantly differentially expressed genes in a single group from the network that contains the top differentially expressed genes in a category. This is done using the Cytoscape API to create subnetworks and provide styles that color nodes based on their differential expression in a given group.

\section{Automation}

Similar to the automation functionality that scNetViz uses, described above, scNetViz, provides its own automation commands, useful for scripts to control scNetViz operations. The following is a list of available commands (details available in the Swagger documentation (Help $\rightarrow$ Automation $\rightarrow$ CyREST Command API)).

\section{List of scNetViz Commands}

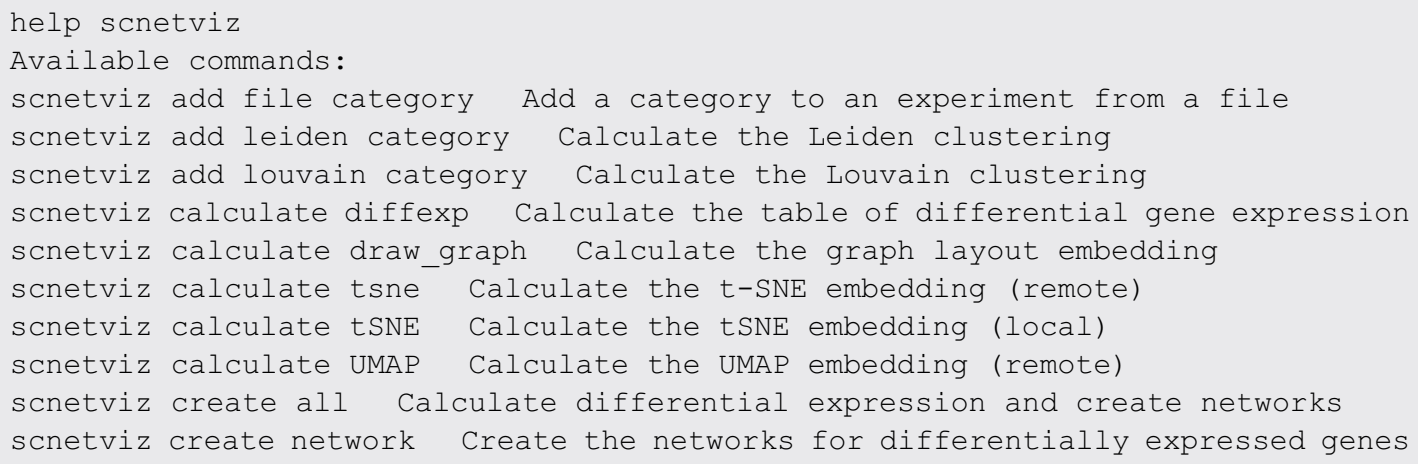




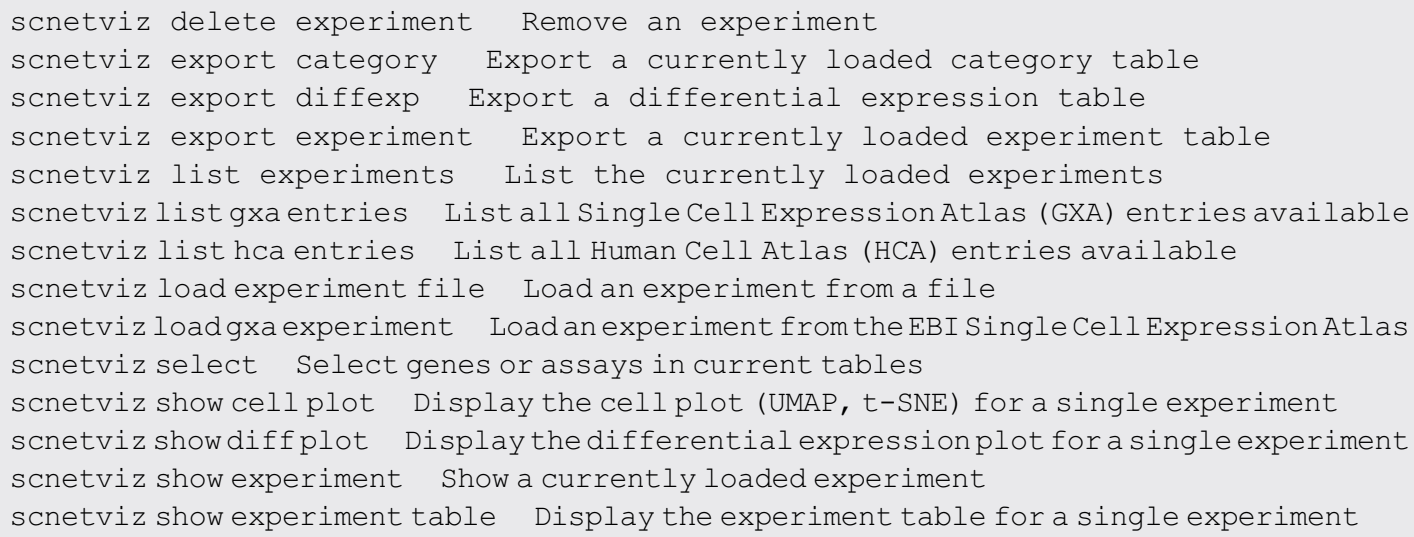

As an example workflow, one might execute the following commands:

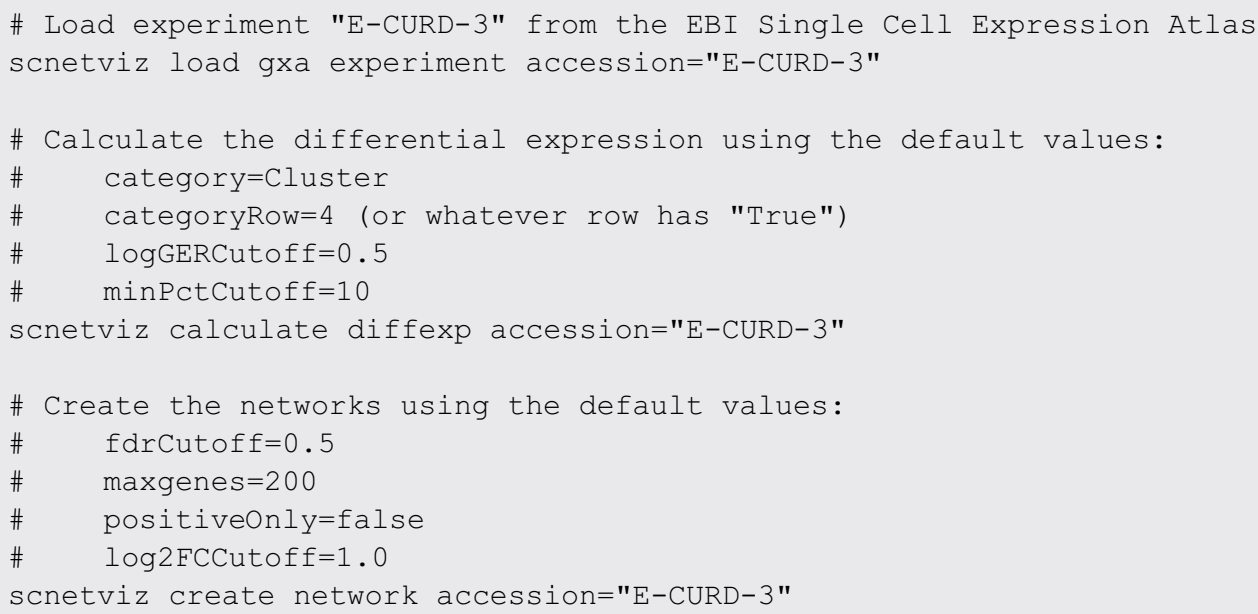

\section{Web service}

As discussed above, the web service component is primarily a wrapper around Scanpy that exposes a limited set of the Scanpy functionality appropriate to the needs of scNetViz. In each case, the web service collects the data and arguments, calls Scanpy pre-processing routines as appropriate, and then calls the Scanpy implementation of the algorithm. By default, the matrix will be normalized, log-transformed, restricted to only the most variable genes, and scaled. The default minimum of 100 genes/cell and a minimum of at least 1 cell for each gene are also used. The parameters to control preprocessing may be changed by opening up the Advanced pre-processing parameters section of the UMAP, t-SNE, Louvain, or Leiden dialogs, which enables the user to change any of the pre-processing defaults.

The web service implements the algorithms using the appropriate scanpy.tl methods (scanpy.tl.tsne, scanpy.tl.umap, scapy.tl.leiden, and scanpy.tl.louvain). In all cases, the matrix is pre-processed and passed to the appropriate Scanpy module. We expose the most useful parameters in each case. For UMAP, we expose the number of neighbors and the minimum distance parameters; for t-SNE, we expose the perplexity, the number of initial dimensions, the early exaggeration parameter and the learning rate. Note that if the number of initial dimensions is set to -1 , a PCA will be calculated first to reduce the dimensionality of the matrix, which can improve the resulting t-SNE embedding. For Leiden and Louvain, we expose the number of neighbors to use to calculate the neighborhood graph before clustering (see scanpy.pp.neighbors). See the Scanpy documentation for a more detailed explanation of the effects of these routines and parameters. 


\section{Use Cases}

We have prioritized providing access to the Single Cell Expression Atlas and Human Cell Atlas. ${ }^{3,4}$ Additionally, researchers can import normalized and clustered scRNA-seq data from local files. The following workflows demonstrate two use cases using either the Single Cell Expression Atlas or local files, and illustrate the utility of scNetViz in integrating network information with scRNA-seq data. Scripted versions of these workflows are also available as R Markdown documents and Jupyter notebooks, which leverage the $\mathrm{RCy} 3^{26}$ and py4cytoscape ${ }^{27}$ packages, respectively. They require Cytoscape (3.7.1 or later), CyREST (3.8.0 or later), and R (version 4.0 or later) or Python (version 3.6 or later). User documentation is available at: https://www.cgl.ucsf.edu/cytoscape/scNetViz/index.shtml. Downloadable notebooks of these workflows are available in $\mathrm{R}$ and Python at http://automation.cytoscape.org.

\section{Use Case \#1: Loading data from the EMBL-EBI Single Cell Expression Atlas for scNetViz analysis}

In this example, we will browse the Single Cell Expression Atlas from within Cytoscape, explore a particular dataset, perform differential expression analysis based on one of the provided cell annotation categories, generate networks from the top differentially expressed genes for each group within the chosen category, and functionally characterize and visualize the networks.

1. Click the $\mathbf{Q}$ icon in the Cytoscape toolbar. This opens the Single Cell Experiment Atlas browser. For illustration, let us select the experiment with Accession E-GEOD-81383, which contains data on three human melanoma cell lines. ${ }^{28}$ This is a dataset of 226 cells from short-term cultures of cell lines derived from subcutaneous metastases. To find this experiment, click the column header labelled Accession and search for E-GEOD-81383 in the resulting table sorted by accession numbers. Select the row with the accession number E-GEOD-81383 by clicking on it. Note that all the steps of this workflow except for functional enrichment analysis can be executed by selecting an experiment and clicking the button labeled Create Networks in the Browse interface. Default parameters for the differential expression analysis and network creation steps can be altered by clicking the gear icon.

2. Click the button labeled View Data. This loads the data and opens an experiment table with three tabs.

a. The Categories tab lists the annotation categories of cells available in the experiment, which represent grouping of cells by cluster labels for this dataset but may represent grouping by cell types or other criteria. Throughout this manuscript, categories refers to cell annotation categories. There are two options listed under Available categories. "Cluster" is the unsupervised grouping of cells computed by the Single Cell Experiment Atlas using the Louvain algorithm ${ }^{29}$ for a range of resolution parameters. Each value of the resolution parameter yields a different number of clusters, which is called the $\mathbf{K}$ value (second column of the displayed table as in Figure 1). In keeping with EBI's practice, by default, scNetViz selects the $\mathbf{K}$ value corresponding to the resolution of 1 for differential analysis and visualization (indicated with a TRUE value corresponding to

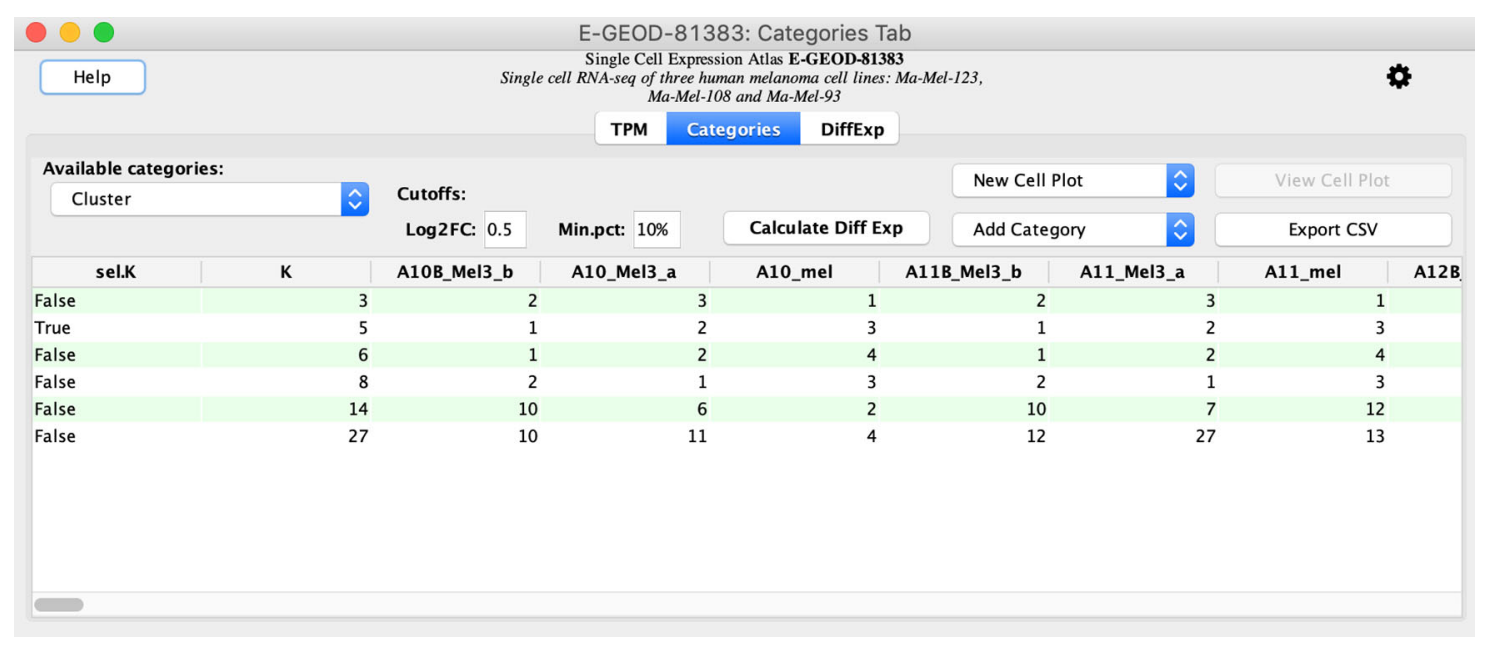

Figure 1. The Categories tab shows the grouping of cells for differential analysis and visualization purposes. The menu labeled New Cell Plot contains options for generating UMAP and t-SNE plots. Note that these results are for data from the Single Cell Expression Atlas release 15 (March, 2021). 


\begin{tabular}{|c|c|c|c|c|c|c|c|c|c|}
\hline \multicolumn{10}{|l|}{00} \\
\hline \multirow[t]{2}{*}{ Help } & \multicolumn{7}{|c|}{$\begin{array}{l}\text { Single Cell Expression Atlas E-GEOD-81383 } \\
\text { Single cell RNA-seq of three human melanoma cell lines: Ma-Mel-123, } \\
\text { Ma-Mel-108 and Ma-Mel-93 }\end{array}$} & \multirow{2}{*}{\multicolumn{2}{|c|}{$\$$}} \\
\hline & & & TPM & Categories & DiffExp & & & & \\
\hline \multicolumn{10}{|l|}{ Comparison: } \\
\hline$k=5$ & & \multirow{2}{*}{\multicolumn{6}{|c|}{ Network analysis: }} & & \\
\hline \multirow{2}{*}{ Each vs. Others } & & & & & & & & View Plots & $\hat{\imath}$ \\
\hline & & FDR: 0 & Log2FC: 1.00 & Max genes: 200 & Positive only & \multicolumn{2}{|c|}{ Create Networks } & Export CSV & \\
\hline Gene & Cluster 1 MTC & Cluster 1 Min.pct & Cluster 1 MDTC & Cluster $1 \log 2 \mathrm{FC}$ & Cluster $1 \mathrm{pValue}$ & Cluster 1 FDR & Cluster 2 MTC & Cluster 2 Min.pct & \\
\hline ENSC00000000003 & 44.049 & $83 \%$ & 60.164 & -.729 & $1.94 \mathrm{E}-2$ & $3.33 \mathrm{E}-2$ & 53.527 & $84 \%$ & \\
\hline ENSC00000000419 & 65.416 & $88 \%$ & 76.318 & .224 & & & 55.716 & $91 \%$ & \\
\hline ENSC00000000457 & 23.146 & $88 \%$ & 31.615 & -.050 & & & 28.581 & $85 \%$ & \\
\hline ENSC000000000460 & 14.276 & $82 \%$ & 25.789 & -1.805 & $1.96 \mathrm{E}-5$ & $9.56 \mathrm{E}-5$ & 58.315 & $78 \%$ & \\
\hline ENSC00000000971 & 5.797 & $59 \%$ & 21.643 & -2.660 & $2.25 \mathrm{E}-5$ & $1.07 E-4$ & 62.984 & $52 \%$ & \\
\hline ENSG000000001036 & 65.186 & $95 \%$ & 70.200 & -.727 & $8.56 \mathrm{E}-2$ & $1.20 \mathrm{E}-1$ & 194.342 & $96 \%$ & \\
\hline ENSC00000001084 & 20.058 & $74 \%$ & 37.442 & -.411 & & & 12.302 & $77 \%$ & \\
\hline ENSC00000001167 & 65.217 & $85 \%$ & 91.304 & .171 & & & 59.523 & $84 \%$ & \\
\hline ENSC00000001460 & 26.560 & $95 \%$ & 28.603 & .983 & $3.21 \mathrm{E}-2$ & $5.10 \mathrm{E}-2$ & 12.870 & $97 \%$ & \\
\hline ENSC00000001461 & 413.697 & $100 \%$ & 413.697 & 2.258 & $1.75 \mathrm{E}-15$ & $1.08 \mathrm{E}-13$ & 116.764 & $94 \%$ & \\
\hline ENSC00000001497 & 61.000 & $72 \%$ & 89.894 & .555 & $4.82 \mathrm{E}-1$ & $5.38 \mathrm{E}-1$ & 34.375 & $76 \%$ & \\
\hline ENSC00000001561 & 3.376 & $8 \%$ & 94.536 & & & & .000 & $9 \%$ & \\
\hline ENSC00000001626 & 2.588 & $52 \%$ & 5.368 & .932 & $3.71 \mathrm{E}-1$ & $4.27 \mathrm{E}-1$ & 1.285 & $58 \%$ & \\
\hline ENSC00000001629 & 101.285 & $89 \%$ & 115.754 & .813 & $9.98 \mathrm{E}-2$ & $1.36 \mathrm{E}-1$ & 49.723 & $92 \%$ & \\
\hline ENSC000000001630 & 164.946 & $99 \%$ & 167.945 & -.538 & $5.73 \mathrm{E}-4$ & $1.72 \mathrm{E}-3$ & 243.851 & $99 \%$ & \\
\hline
\end{tabular}

Figure 2. The DiffExp tab allows selection of cutoffs for genes to be used for network analysis and contains options for generating gene by cell heatmaps and violin plots showing gene expression distributions across cell annotation categories.

the selected $\mathbf{K}$ in the column titled sel.K; see Figure 1). ${ }^{30}$ The other columns show single cell identifiers and their cluster memberships. To get the table of differentially expressed genes press the button labeled Calculate Diff Exp. This initiates a comparison of each cluster for $\mathrm{K}=5$ with other clusters.

b. The results are displayed in the DiffExp tab. Click the Create Networks button. This fetches protein-protein networks for the proteins encoded by genes that satisfy the Network Analysis cutoffs based on FDR (maximum false discovery rate), Log2FC (minimum absolute value of log base 2 of the fold change in expression), and Max genes (maximum number of selected proteins; see Figure 2).

3. The networks are listed in the Control Panel (left side) and individually displayed in the main Cytoscape Desktop window (Figure 3). There are six networks, one for each of the five clusters and one for all the clusters collectively. Select the part of the network that is of interest by holding down the left mouse button on the canvas background and dragging the mouse while holding down the Shift or Ctrl key (Command on Mac).

4. Along the right edge of the Cytoscape window in the Results Panel, click on the STRING tab (see Figure 3). Click on the button labeled Functional Enrichment to retrieve the enriched pathways and ontology terms in the selected part of the network. The results are displayed in the STRING Enrichment tab in the Node Table at the bottom. They can be filtered for, say, pathway terms using the Filter enrichment table option located on the top left of the tab and represented with a black funnel icon.

Use Case \#2: Loading your own scRNA-Seq data from local files for scNetViz analysis

In this example, we will import normalized scRNA-seq data and cluster assignments from local files, visualize all cells in a UMAP plot, perform differential expression analysis based on one of the provided categories, visualize as a combined gene expression heat map, and generate networks for the most significantly differentially expressed genes from each group within the chosen category.

1. Choose Apps $\rightarrow \mathbf{s c N e t V i z} \rightarrow$ Load Experiment $\rightarrow$ Import from file from the menu, then browse to locate and open a zip, tar.gz, tgz, or gzip file of the three MatrixMarket files (.mtx, .mtx_cols, .mtx_rows) comprising a normalized scRNA-seq quantification dataset. These files can be obtained by normalizing standard outputs from an scRNA-seq processing software such as CellRanger. ${ }^{31}$ For illustration, we use an experiment on 329 cells at different time points of differentiation starting from human embryonic stem cells (download the normalized counts files and clustering results for accession ID E-GEOD-109979 from the Single Cell Expression Atlas ${ }^{32}$ ). In the dialog, select the species, which is Homo sapiens for this data. 

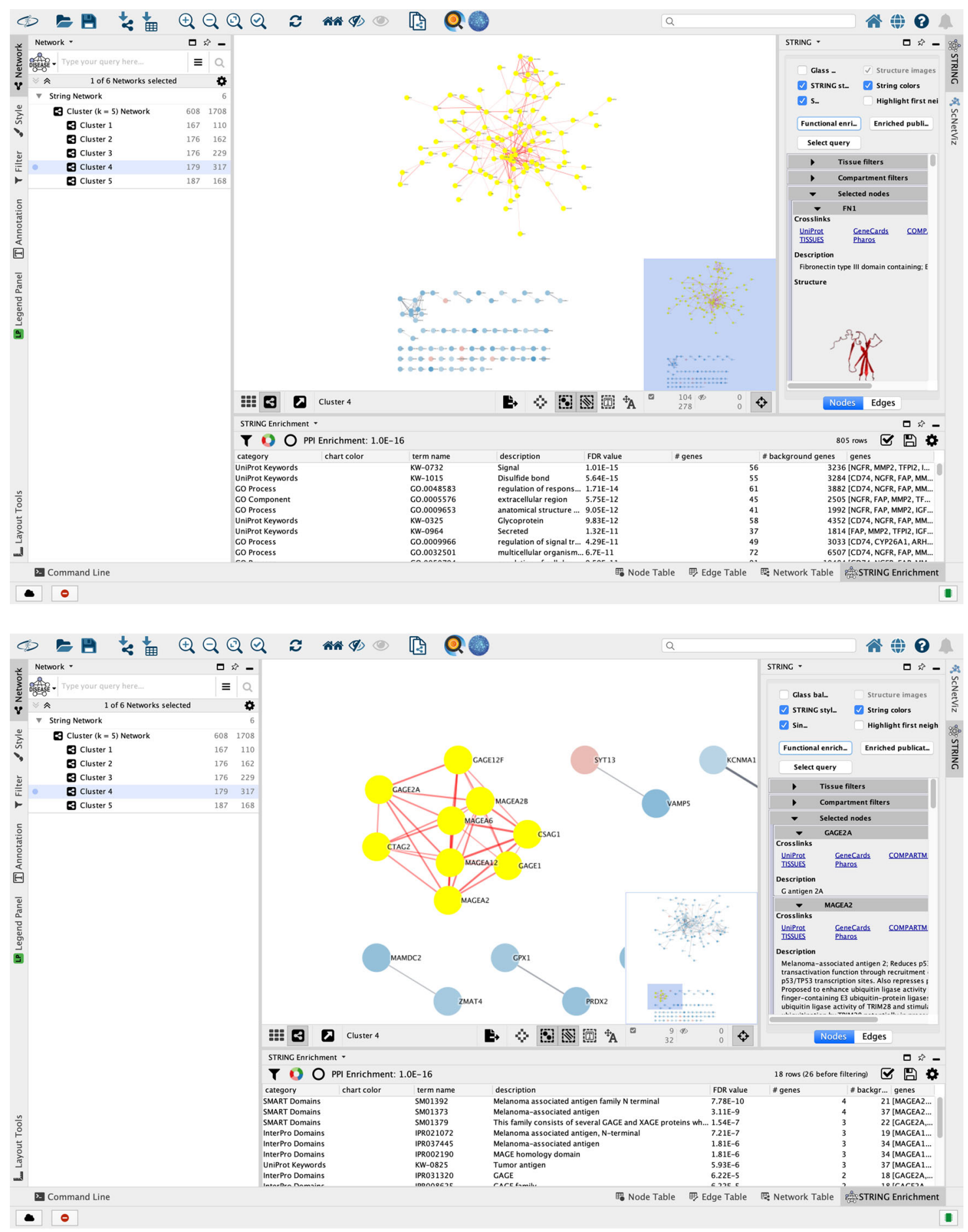

Figure 3. The networks from the STRING database are displayed in the main Cytoscape window. The illustration shows the interaction network for genes specific to the group labeled "Cluster 4". Users can filter the complete network using the network analysis tools in Cytoscape. (a) A selection of the largest connected component, which is highlighted with yellow nodes and red edges. (b) Other sub-networks might also be of interest. The second-largest connected component of Cluster 4 shows enrichment of melanoma-associated terms, which is consistent with the fact that this experiment is on melanoma cell lines. 


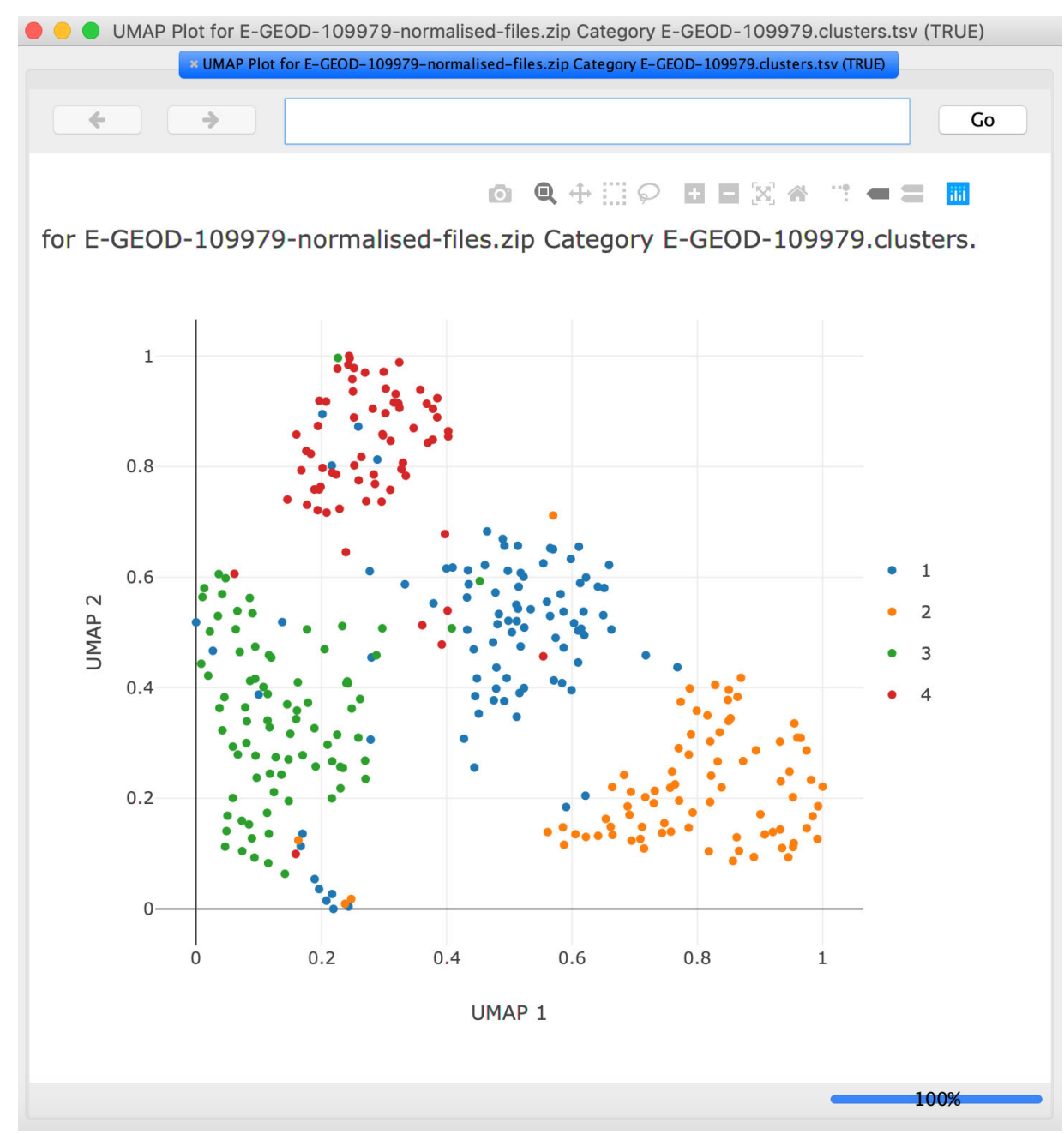

Figure 4. The UMAP plot is displayed in a pop-up window and can be explored interactively. Each dot is a cell, and coloring is by cluster number. Note that the illustration shows results obtained from analysis with the default settings.

2. From the window with the experiment table, load the downloaded clustering results as category data using the Import from file option in the menu under Add Category. Alternatively, select the option for Louvain clustering or Leiden clustering from this menu to perform unsupervised clustering of the cells.

3. From the Categories tab, select the row with the grouping of cells that should be highlighted on the UMAP plot. Select the UMAP option from the New Cell Plot menu. If required, change the options and press OK to view the UMAP plot (Figure 4).

4. Switch to the Categories tab on the experiment table window. Select a category by clicking its row and press Calculate Diff Exp. This switches the display to the Diff Exp tab.

5. Select the Heatmap option from the View Plots menu on the Diff Exp tab. This generates a gene expression heatmap for genes that meet the filtering criteria (Figure 5).

6. Click the Create Networks button on the Diff Exp tab to fetch the protein functional interaction networks for the top genes. 


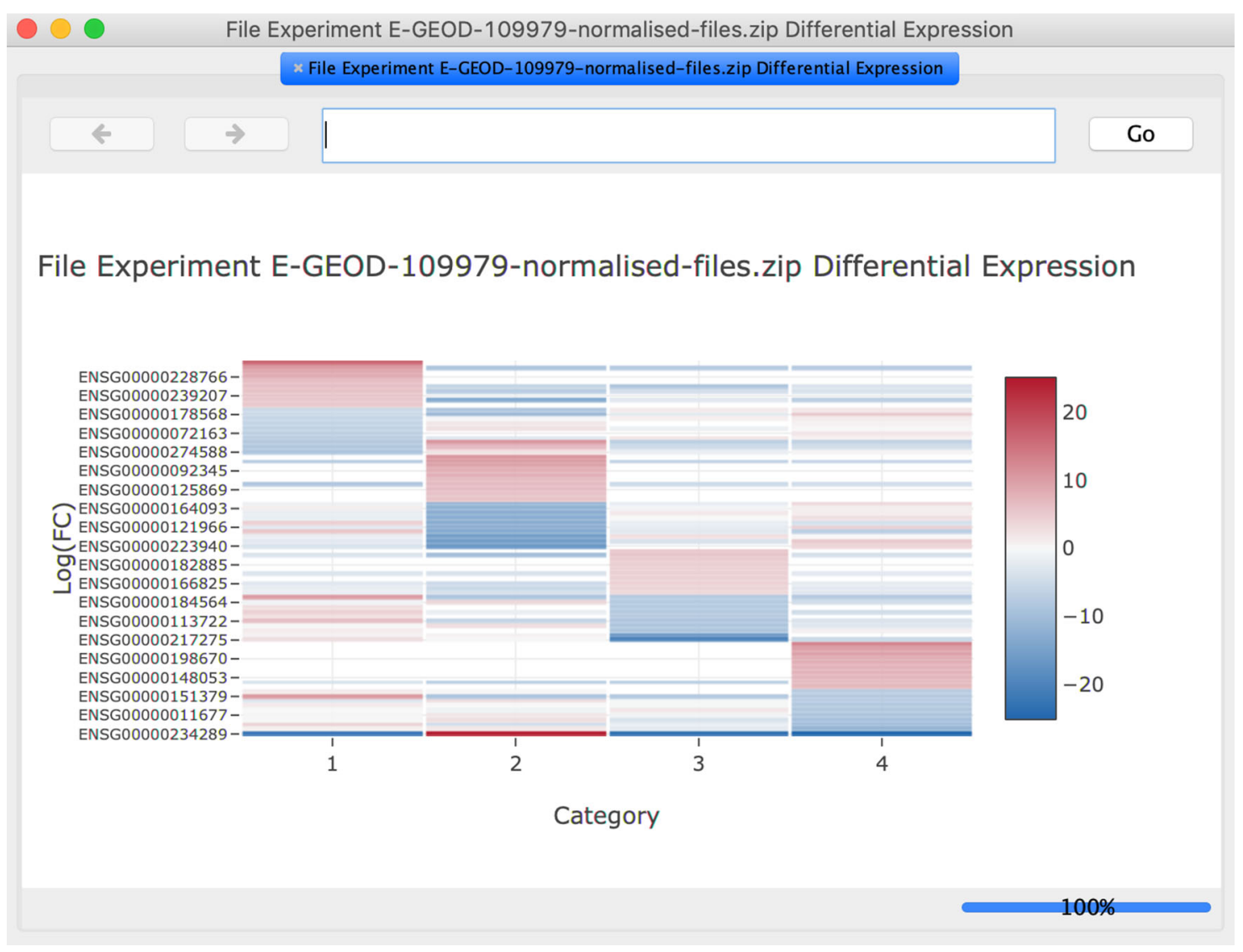

Figure 5. The heatmap for the top differentially expressed genes is displayed in a pop-up window and can be explored interactively. Note that the illustration shows results obtained from analysis with the default settings.

\section{Conclusion}

scNetViz is a Cytoscape app for identifying differentially expressed genes characteristic of cell clusters and displaying networks of the corresponding proteins for further analysis. This workflow aids interpretation of cell clusters from scRNA-seq and related single-cell resolution data. It provides several ways of plotting cells and gene expression data, e.g., heatmap, violin, t-SNE, and UMAP plots. We describe two use cases demonstrating its utility for integrating network information with local or public single-cell RNA sequencing data. Users can set the analysis parameters, save settings, analyze their own data or load data from public atlases, and interactively select gene sets or cell clusters for network analysis. scNet Viz is highly portable to diverse computational systems via its GUI or CyREST services. It only has Cytoscape and its stringApp, cyBrowser, cyPlot and enhancedGraphics apps as dependencies. Compared with other single cell analysis tools it provides a user-friendly interface and no coding expertise is needed for its use.

Currently, scNetViz does not have options to normalize or batch-correct raw counts. To analyze their own data, users must input normalized and batch corrected data. The methods for normalization, batch correction, clustering, and differential expression analysis are under active development and benchmarking. As the standard workflow of analysis evolves, we will continue to integrate newer methods in scNetViz. For differential analysis, scNetViz only supports comparing a group of cells within a category with the set of cells in all other groups in that category combined (e.g. to identify cell-typespecific genes, or gene expression markers). In the future, we will support more general comparisons, including comparison of any two groups of cells provided or selected by the user. Future development will also enable excluding pseudogenes or other gene sets from the analysis. File formats are also evolving. For instance, the Loom format is becoming more popular. This format is not supported by scNetViz currently. As new standards are adopted for single-cell data, we will add support for them. 


\section{Software availability}

- Software available from the Cytoscape App Store: https://apps.cytoscape.org/apps/scnetviz

- Software code available from GitHub: https://github.com/RBVI/scNetViz

- Archived source code at the time of publication: https://doi.org/10.5281/zenodo. $4641480^{33}$

- License: Apache License, Version 2.0

- Online manual: https://www.cgl.ucsf.edu/cytoscape/scNetViz/index.shtml

- scNetViz Rmd and Python notebooks: http://automation.cytoscape.org

\section{Data availability}

The source data used for the use cases are available from Single Cell Expression Atlas.

- Use Case \#1: https://www.ebi.ac.uk/gxa/sc/experiments/E-GEOD-81383/downloads

- Use Case \#2: https://www.ebi.ac.uk/gxa/sc/experiments/E-GEOD-109979/downloads

\section{Acknowledgements}

We thank Kristina Hanspers and Yihang Xin for testing the $s c N e t V i z$ Rmd and Python notebooks and providing feedback.

1. Tanay A, Regev A: Scaling single-cell genomics from phenomenology to mechanism. Nature. 2017; 541(7637): 331-338.

PubMed Abstract | Publisher Full Text | Free Full Text

2. Luecken MD, Theis FJ: Current best practices in single-cell RNAseq analysis: a tutorial. Mol Syst Biol. 2019; 15(6): e8746. PubMed Abstract | Publisher Full Text | Free Full Text

3. Regev A, Teichmann SA, Lander ES, et al.: Science forum: the human cell atlas. Elife. 2017; 6: e27041.

PubMed Abstract | Publisher Full Text | Free Full Text

4. Papatheodorou I, Moreno P, Manning J, et al.: Expression Atlas update: from tissues to single cells. Nucleic Acids Res. 2020; 48(D1): D77-D83.

PubMed Abstract | Publisher Full Text | Free Full Text

5. Amezquita RA, Lun ATL, Becht E, et al.: Orchestrating single-cell analysis with Bioconductor. Nat Methods. 2020; 17(2): 137-145. PubMed Abstract | Publisher Full Text | Free Full Text

6. Blencowe $M$, Arneson D, Ding J, et al.: Network modeling of singlecell omics data: challenges, opportunities, and progresses. Emerg Top Life Sci. 2019; 3(4): 379-398. PubMed Abstract | Publisher Full Text | Free Full Text

7. Cha J, Lee I: Single-cell network biology for resolving cellular heterogeneity in human diseases. Exp Mol Med. 2020; pages 1-11. PubMed Abstract | Publisher Full Text

8. Lee LY, Pandey AK, Maron BA, et al.: Network medicine in cardiovascular research. Cardiovasc Res. 2020. PubMed Abstract | Publisher Full Text

9. Shi $\mathrm{H}$, Yan K-K, Ding $\mathrm{L}$, et al.: Network approaches for dissecting the immune system. Iscience. 2020; page 101354. PubMed Abstract | Publisher Full Text | Free Full Text

10. Iacono G, Massoni-Badosa R, Heyn H: Single-cell transcriptomics unveils gene regulatory network plasticity. Genome Biol. 2019; 20(1): 110.

PubMed Abstract | Publisher Full Text | Free Full Text

11. Kamimoto K, Hoffmann CM, Morris S: CellOracle: Dissecting cell identity via network inference and in silico gene perturbation. bioRxiv. 2020.

Publisher Full Text
12. Turki T, Yh T: SCGRNs: Novel supervised inference of single-cell gene regulatory networks of complex diseases. Comput Biol Med. 2020; 118: 103656. PubMed Abstract | Publisher Full Text

13. Li WV, Li Y: Inferring sparse gene co-expression networks from single-cell expression data. bioRxiv. 2020. Publisher Full Text

14. Mohammadi S, Davila-Velderrain J, Kellis M: Reconstruction of celltype-specific interactomes at single-cell resolution. Cell Syst. 2019; 9(6): 559-568. PubMed Abstract | Publisher Full Text | Free Full Text

15. Szklarczyk D, Gable AL, Lyon D, et al.: STRING v11: protein-protein association networks with increased coverage, supporting functional discovery in genome-wide experimental datasets. Nucleic Acids Res. 2019; 47(D1): D607-D613. PubMed Abstract | Publisher Full Text | Free Full Text

16. Shannon P, Markiel A, Ozier O, et al.: Cytoscape: a software environment for integrated models of biomolecular interaction networks. Genome Res. 2003; 13(11): 2498-2504 PubMed Abstract | Publisher Full Text | Free Full Text

17. Otasek D, Morris JH, Bouças J, et al.: Cytoscape automation: empowering workflow-based network analysis. Genome Biol. 2019; 20(1): 1-15. PubMed Abstract | Publisher Full Text | Free Full Text

18. Doncheva NT, Morris JH, Gorodkin J, et al.: Cytoscape StringApp: network analysis and visualization of proteomics data. J Proteome Res. 2018; 18(2): 623-632. PubMed Abstract | Publisher Full Text | Free Full Text

19. Yih A, Baker L, Magee L, et al.: cyPlot: A utility to display data plots. Reference Source

20. Morris JH, Kuchinsky A, Ferrin TE, et al.: enhancedGraphics: a Cytoscape app for enhanced node graphics. F1000Res. 2014; 3. PubMed Abstract | Publisher Full Text | Free Full Text

21. McInnes L, Healy J, Melville J: Umap: Uniform manifold approximation and projection for dimension reduction. arXiv preprint arXiv:1802.03426. 2018

22. van der Maaten L, Hinton G: Visualizing data using t-SNE. J Mach Learn Res. 2008; 9(Nov): 2579-2605. 
23. Alexander Wolf $F$, Angerer $P$, Theis FJ: SCANPY: large-scale singlecell gene expression data analysis. Genome Biol. 2018; 19(1): 1-5. PubMed Abstract | Publisher Full Text | Free Full Text

24. Matrix Market: File formats. Reference Source

25. Ono K, Muetze T, Kolishovski G, et al.: Turbocharging cytoscape access for external tools via a RESTful API. F1000Res. 2015; 4 PubMed Abstract | Publisher Full Text | Free Full Text

26. Gustavsen JA, Pai S, Isserlin R, et al.: RCy3: network biology using cytoscape from within R. F1000Res. 2019; 8. PubMed Abstract | Publisher Full Text | Free Full Text

27. Ono K, Bouças J, Nishida K, et al.: py4cytoscape. Reference Source

28. Gerber T, Willscher E, Loeffler-Wirth $\mathrm{H}$, et al.: Single cell RNA-seq of three human melanoma cell lines: Ma-Mel-123, Ma-Mel-108 and Ma-Mel-93.

Reference Source
29. Blondel VD, Guillaume J-L, Lambiotte R, et al.: Fast unfolding of communities in large networks. J Stat Mech: Theory Experiment 2008; 2008(10): P10008.

Publisher Full Text

30. Single Cell Expression Atlas help. Reference Source Accessed date: March 11, 2021.

31. Zheng GXY, Terry JM, Belgrader $P$, et al.: Massively parallel digital transcriptional profiling of single cells. Nat Commun. 2017; 8(1): $1-12$.

PubMed Abstract | Publisher Full Text | Free Full Text

32. Lu J, Baccei A, da Rocha EL, et al.: Single-cell RNA-seq of human $\mathrm{H} 9$ cells undergoing definitive endoderm differentiation. Reference Source

33. Morris S, Pico A, mohamedzak: RBVI/scNetViz: v1.2.0 (Version v1.2.0). Zenodo. 2021, March 26. Publisher Full Text 


\section{Open Peer Review}

\section{Current Peer Review Status:}

\section{Version 1}

Reviewer Report 15 November 2021

https://doi.org/10.5256/f1000research.55741.r98565

(C) 2021 Kamimoto K. This is an open access peer review report distributed under the terms of the Creative Commons Attribution License, which permits unrestricted use, distribution, and reproduction in any medium, provided the original work is properly cited.

\section{Kenji Kamimoto}

Department of Developmental Biology, Washington University School of Medicine in St. Louis, St. Louis, MO, USA

The authors have developed scNetViz, a Cytoscape-based software for constructing and analyzing cluster-specific protein-protein interaction networks from scRNA-seq data.

scNetViz selects genes differentially expressed in each cluster based on scRNA-seq data and constructs a cluster-specific network by filtering the universal protein-protein interaction network registered in the STRING database. The system includes all necessary steps, including data search, download, clustering, network filtering, and visualization. All these operations can be performed with the GUI, enabling easy and intuitive network analysis.

The installation of scNetViz by Cytoscape's App Manager is simple, and I could install scNetViz without any trouble. The use cases presented in the paper help the reader to understand the typical usage of scNetViz, and they are described clearly. The analysis with a Single Cell Expression Atlas could be conducted without any problem.

scNetViz creates a network by filtering the STRING protein-protein interaction network database. Although this concept is described in the introduction and later sections, it is not clear from the title and abstract of the paper (version 1). Thus, the title and abstract might make the wrong impression that scNetViz can infer a de novo gene regulatory network from scRNA-seq data rather than filtering the pre-existing network. Therefore, to clarify the concept of the scNetViz paper, I would like to recommend adding descriptions of the following points in the abstract or title.

1. scNetViz is a network filtering analysis, not a de novo GRN inference algorithm.

2. scNetViz uses protein-protein interaction network analysis, and it is not another GRN analysis such as co-expression or transcriptional gene regulatory network.

\section{Is the rationale for developing the new software tool clearly explained?}


Is the description of the software tool technically sound?

Yes

Are sufficient details of the code, methods and analysis (if applicable) provided to allow replication of the software development and its use by others?

Yes

Is sufficient information provided to allow interpretation of the expected output datasets and any results generated using the tool?

Yes

Are the conclusions about the tool and its performance adequately supported by the findings presented in the article?

Yes

Competing Interests: No competing interests were disclosed.

Reviewer Expertise: Computational Biology

I confirm that I have read this submission and believe that I have an appropriate level of expertise to confirm that it is of an acceptable scientific standard.

Author Response 19 Jan 2022

Krishna Choudhary

We thank the reviewer for their time and thoughtful comments. While the difference between de novo GRN inference and networking filtering analysis is important to understand, it may cause more confusion than clarification to highlight this unrelated algorithm in the title and abstract. Hence, we believe that this detail is best described with literature references in the introduction where more space can be given to background and contextual topics. Perhaps we can specify "protein-protein interaction networks" in a future version of the abstract.

Competing Interests: No competing interests were disclosed.

Reviewer Report 26 July 2021

https://doi.org/10.5256/f1000research.55741.r86919

(C) 2021 Pinello L. This is an open access peer review report distributed under the terms of the Creative Commons Attribution License, which permits unrestricted use, distribution, and reproduction in any medium, provided the original work is properly cited.

Luca Pinello 
Molecular Pathology Unit, Center for Cancer Research, Massachusetts General Hospital, Department of Pathology, Harvard Medical School, Boston, MA, USA

Choudhary et al., present scNetViz a useful Cytoscape app to perform network analysis on singlecell RNA-seq data at cluster level.

The manuscript is well written and clear. The software is well documented with clear examples, use cases and nice illustrative figures.

Particularly helpful is the possibility to load datasets from several public repositories (EMBL-EBI and Human Cell Atals) with simple dedicated functions.

Some points to consider to improve the manuscript and software:

1. The documentation regarding the installation of the software. For example on OSX it is very tricky to install and use it since Java applications that are not signed are not allowed and a warning message is displayed. This may discourage several users, so it could be helpful to have step by step and explicit instructions that cover this case and also that assume a user doesn't have yet Cytoscape installed. On Windows 10 things are much easier and I was able to install it by using the Apps-> App Manager... function.

2. The search text are on the window "Single cell Expression Atlas Browser" doesn't work properly and I was not able to select E-GEOD-8138 with this function (it doesn't scroll automatically to the correct line).

3. I was able to reproduce the analysis for E-GEOD-81383, however the tool failed to create a network for E-CURD-3 when clicking "Create Networks " (the first dataset presented on the provided list). If not all the datasets are supported, it would be helpful showing to the users only the ones where it is possible to create networks and explaining why it is not possible to build networks for some datasets.

Is the rationale for developing the new software tool clearly explained?

Yes

Is the description of the software tool technically sound?

Yes

Are sufficient details of the code, methods and analysis (if applicable) provided to allow replication of the software development and its use by others?

Partly

Is sufficient information provided to allow interpretation of the expected output datasets and any results generated using the tool?

Yes

Are the conclusions about the tool and its performance adequately supported by the findings presented in the article? 
Yes

Competing Interests: No competing interests were disclosed.

Reviewer Expertise: Computational Biology

I confirm that I have read this submission and believe that I have an appropriate level of expertise to confirm that it is of an acceptable scientific standard.

Author Response 19 Jan 2022

Krishna Choudhary

We thank the reviewer for their time and thoughtful comments. We have addressed all the comments below.

1. The documentation regarding the installation of the software. For example on OSX it is very tricky to install and use it since Java applications that are not signed are not allowed and a warning message is displayed. This may discourage several users, so it could be helpful to have step by step and explicit instructions that cover this case and also that assume a user doesn't have yet Cytoscape installed. On Windows 10 things are much easier and I was able to install it by using the Apps-> App Manager... function.

Response: Thank you for testing the installation of scNetViz on OSX and Windows platforms. Since scNetViz is one of many Cytoscape apps featured in this app article collection, we are assuming Cytoscape installation and familiarity. We recommend users to get the latest installation instructions directly from Cytoscape.org (and that is good because the instructions may change over time).

2. The search text are on the window "Single cell Expression Atlas Browser" doesn't work properly and I was not able to select E-GEOD-8138 with this function (it doesn't scroll automatically to the correct line).

Response: The search works by highlighting all lines that contain the search string. Since there can be multiple result lines (e.g., when searching for a species), we did not design a scroll behavior. It is working as designed. However, based on your feedback, we will work on a new feature to either scroll to the first hit or filter the table for hits in order to improve the display of search results.

3. I was able to reproduce the analysis for E-GEOD-81383, however the tool failed to create a network for E-CURD-3 when clicking "Create Networks " (the first dataset presented on the provided list). If not all the datasets are supported, it would be helpful showing to the users only the ones where it is possible to create networks and explaining why it is not possible to build networks for some datasets.

Response: Thank you for finding this bug. We could reproduce the error. The issue is related to the species (Plasmodium falciparum) not being recognized. We have opened a bug report to characterize the issue more thoroughly in order to either fix it or detect it ahead of time 
(like you suggested).

Competing Interests: No competing interests were disclosed.

The benefits of publishing with F1000Research:

- Your article is published within days, with no editorial bias

- You can publish traditional articles, null/negative results, case reports, data notes and more

- The peer review process is transparent and collaborative

- Your article is indexed in PubMed after passing peer review

- Dedicated customer support at every stage

For pre-submission enquiries, contact research@f1000.com 